\title{
Contrasting the beam interaction characteristics of selected lasers with a partially stabilised zirconia (PSZ) bio-ceramic
}

\author{
J. Lawrence \\ Manufacturing Engineering Division, School of Mechanical \& Production Engineering, Nanyang \\ Technological University (NTU), Nanyang Avenue, Singapore 639798.
}

\section{Correspondence}

Dr. Jonathan Lawrence

Manufacturing Engineering Division,

School of Mechanical \& Production Engineering,

Nanyang Technological University (NTU),

Nanyang Avenue,

Singapore 639798.

Tel : (+65) 67905542

Fax : (+65) 67911859

e-mail : mjlawrence@ntu.edu.sg 


\begin{abstract}
Differences in the beam interaction characteristics of a $\mathrm{CO}_{2}$ laser, a Nd:YAG laser, a high power diode laser (HPDL) and an excimer laser with a partially stabilised zirconia (PSZ) bio-ceramic have been studied. A derivative of Beer-Lambert's law was applied and the laser beam absorption lengths of the four lasers were calculated as $33.55 \times 10^{-3} \mathrm{~cm}$ for the $\mathrm{CO}_{2}$ laser, $18.22 \times 10^{-3} \mathrm{~cm}$ for the Nd:YAG laser, $17.17 \times 10^{-3} \mathrm{~cm}$ for the HPDL and $8.41 \times 10^{-6} \mathrm{~cm}$ for the excimer laser. It was determined graphically that the fluence threshold values at which significant material removal was effected by the $\mathrm{CO}_{2}$ laser, the Nd:YAG laser, the HPDL and the excimer laser were $52 \mathrm{~J} / \mathrm{cm}^{2}$, $97 \mathrm{~J} / \mathrm{cm}^{2}, 115 \mathrm{~J} / \mathrm{cm}^{2}$ and $0.48 \mathrm{~J} / \mathrm{cm}^{2}$ respectively. The thermal loading value for the $\mathrm{CO}_{2}$ laser, the $\mathrm{Nd}$ :YAG laser, the HPDL and the excimer laser were calculated as being $1.55 \mathrm{~kJ} / \mathrm{cm}^{3}, 5.32 \mathrm{~kJ} / \mathrm{cm}^{3}$, $6.69 \mathrm{~kJ} / \mathrm{cm}^{3}$ and $57.04 \mathrm{~kJ} / \mathrm{cm}^{3}$ respectively.
\end{abstract}

Keywords: Laser; Partially stabilised zirconia (PSZ); Bio-ceramic; Interaction characteristics; Threshold fluence; Thermal loading 


\section{Introduction}

There is undoubtedly much global interest in advancing medical science and technology in the area of biomaterials. Consequently, work to this end is often at the forefront of research in many disciplines, with laser materials processing being no exception. Yet despite this, to date only an extremely limited amount of work pertaining to the use of lasers for treating and processing biomaterials has been published. To improve the corrosion characteristics of NiTi shape memory alloys (SMAs) in physiological solutions, Villermaux et al [1] examined the possibility of treating the surface of the SMAs with excimer laser radiation. The study concluded that the corrosion resistance of the material was increased due to homogenisation of the surface as a result of melting, surface hardening and the thickening of the surface oxide layer. Work by Guzzardella et al [2] found that low power diode laser (LPDL) treatment of implanted prosthetic bone devices fabricated from hydroxyapatite (HA) brought about considerable enhancement in the bone-HA implant interface. Using $\mathrm{CO}_{2}, \mathrm{Nd}: \mathrm{YAG}$ excimer and high power diode laser (HPDL) radiation Lawrence and Li found that it was possible to effect changes in the wettability characteristics of the bio-polymers polymethyl methacrylate (PMMA) [3] and polyethylene (PE) [4].

But, if the laser is to open up entirely new technologies and unique application areas within the biomaterials field then the fundamental differences in the beam absorption characteristics by materials that exist between different lasers must be investigated. This is particularly true for the relatively contemporary HPDL. Such studies are necessary as the outcomes of many laser processing procedures are inextricably influenced by this parameter. For direct surgical applications this implication has been realised and consequently, many studies have been carried out to examine the effects of laser wavelength variation [5-11]. These studies have revealed clear differences in the performance and effectiveness of many different lasers when interacting with various body parts. In contrast, comparative investigations of the differences in the beam interaction characteristics of the predominant materials processing lasers, the $\mathrm{CO}_{2}$, the Nd:YAG and the excimer laser, are few in number [12-15]. Even fewer in number are practical comparisons between the established materials processing lasers and the recently arrived HPDL. Previously Schmidt et al [16] compared the performance of $\mathrm{CO}_{2}$, excimer and HPDLs in the removal of chlorinated rubber coatings from concrete surfaces and remarked on wavelength dependant differences in the process performance. Also, Bradley et al [17] compared the $\mathrm{CO}_{2}$ and HPDL for the treatment of $\mathrm{Al}_{2} \mathrm{O}_{3}$-based refractory materials in terms of microstructure and observed wavelength dependant microstructural 
characteristics unique to each laser. In more comprehensive investigations, Lawrence and $\mathrm{Li}$ compared the effects of $\mathrm{CO}_{2}, \mathrm{Nd}: \mathrm{YAG}$, excimer and HPDL radiation on the wettability characteristics of ceramic [18], metallic $[19,20]$ and polymeric $[3,4]$ materials, noting that changes in the wettability characteristics of the material varied depending upon the laser type. Furthermore, Lawrence et al carried out a comparative study of the characteristic of the glazes generated on the surface of concrete with $\mathrm{CO}_{2}$ and HPDL's [21, 22], as well determining the absorption depths of $\mathrm{CO}_{2}$ and HPDL beams in ordinary Portland cement $[23,24]$ and an $\mathrm{Al}_{2} \mathrm{O}_{3}$-based refractory [25].

By using a $\mathrm{CO}_{2}, \mathrm{Nd}$ Y YAG, excimer and a HPDL this paper aims to provide comparative information on the absorption characteristics (in terms of laser wavelength variations) of a partially stabilised zirconia (PSZ) bio-ceramic through such attributes as absorption length and thermal loading. PSZ is a ceramic that has found wide usage in medical and dental surgery. Within medicine it is commonly used to fabricate hip ball joints, whilst in dentistry it is used to manufacture dental implants, dental posts, brackets and inlays. Consequently, it is believed that this work will provide valuable insight into some of the performance characteristics of the HPDL in comparison with the other more traditional materials processing lasers for the treatment of biomaterials, in particular PSZ.

\section{Experimental procedures}

Four industrial lasers were used in this work: a $\mathrm{CO}_{2}$ laser (Rofin-Sinar, RS-3000), a Nd:YAG laser (Lumonix, JK704) a KrF excimer laser (Lambda Physik, 200mc) and a GaAlAs HPDL (Rofin-Sinar, DL 015). The general operating characteristics of the lasers used in the study are detailed in Table1. The $\mathrm{CO}_{2}$ laser beam was delivered to the surface of the PSZ bio-ceramic samples by focusing the beam through a $150 \mathrm{~mm}$ focal length $\mathrm{ZnSe}$ lens to give a stable diverging beam with beam diameters of 0.6-1.8 mm. The HPDL beam used ranged in size from 1.6- $2.1 \mathrm{~mm}$ in diameter and was delivered to the surface of the PSZ bio-ceramic samples by means of a $5 \mathrm{~m}$ long, $1.5 \mathrm{~mm}$ core diameter optical fibre, the end of which was connected to a $42 \mathrm{~mm}$ focal length lens assembly. The beams produced by both the $\mathrm{CO}_{2}$ laser and the HPDL were multi-mode. For the Nd:YAG laser, the beam was delivered to the surface of the PSZ bio-ceramic samples by means of a $4 \mathrm{~m}$ long, $1 \mathrm{~mm}$ core diameter optical fibre. The beam, which was of a Gaussian profile, was then focused on to the surface of the PSZ bio-ceramic samples by means of a $120 \mathrm{~mm}$ focal length focusing lens assembly with diameters of 1.2-2.2 $\mathrm{mm}$. In the case of the Nd:YAG and HPDLs the focussing optics assembly units were attached to the z-axis of a 3-axis computerised numerical control (CNC) gantry table, whilst the PSZ 
bio-ceramic samples were placed on the x-axis. In the $\mathrm{CO}_{2}$ laser arrangement the focussing optics were fixed and the PSZ bio-ceramic samples were placed on the $\mathrm{x}$-axis of a CNC x-y table. In all three instances the laser optics were protected by means of a coaxially blown Ar shield gas jet a rate of $5 \mathrm{l} / \mathrm{min}$. The excimer laser radiation was firstly passed directly through a circular aperture and then a beam homogenizer. The beam was then focussed on the surface of the PSZ bio-ceramic samples to a spot size of $0.5 \times 0.5 \mathrm{~mm}^{2}$ to $3.5 \times 3.5 \mathrm{~mm}^{2}$. Here the PSZ bio-ceramic samples were attached using double-sided tape to the $\mathrm{x}$-axis of a CNC x-y table. The whole arrangement was tipped vertically so as to be perpendicular the incident excimer laser beam. The excimer laser beam had a square top hat profile and the processing took place in an air atmosphere.

For the purpose of experimental convenience the PSZ bio-ceramic was fabricated into blocks $(25 \times 25$ x $5 \mathrm{~mm}$ ) prior to laser treatment. The PSZ bio-ceramic was treated with all four lasers at room temperature and in normal atmospheric conditions. Experiments were carried out in order to determine the minimum threshold fluence, the absorption length and to examine the thermal loading characteristics of the PSZ bio-ceramic. Here it was necessary that the fluences of all four lasers were set such that no melting occurred. The absence of melting and vitrification was verified by subjecting the irradiated areas to an X-ray diffraction (XRD) analysis to ensure the PSZ bio-ceramic retained its crystallinity.

\section{Results and discussion}

The depth of material removed per pulse or shot, $h$, for organic polymer, inorganic insulating and semi-conducting materials has been frequently described by a logarithmic law which follows, heuristically, from Beer-Lambert's law [26]

$$
h=\frac{1}{\alpha} \ln \left(\frac{F}{F_{t}}\right)
$$

where $\alpha$ is the optical absorption coefficient, $F$ is the laser fluence and $F_{t}$ is the threshold value of the fluence at which significant material removal occurs. Moreover, this expression has been shown to be completely applicable to other composite and ceramic materials [23-25]. It is important to realise that the validity of (1) rests on the assumption that the plume species resulting from material removal absorb the laser beams in a similar manner to the solid. Also, probable changes in the absorption level of the material as removal progresses are not taken in account by (1). Nonetheless, as the work of 
Andrew et al [27], Schmidt et al [16] and Lawrence et al [23-25] demonstrated, this simple form of the expression is quite adequate as a first approximation. Also, according to Bäuerle [26] the expression is applicable to ablation processes which are either photophysical or photochemical in nature. It is therefore acceptable to use the expression in instances such as this, where laser wavelengths, and consequently interaction mechanisms, vary considerably (from UV to IR).

The relationship between $h$ and $F$ for the four laser beams incident with the PSZ bio-ceramic are given in Figs. 1, 2, 3 and 4. As one can see from these plots, $h$ for the PSZ bio-ceramic irradiated with all four lasers was seen to display a logarithmic dependence on $F$. Further, by using an optical profiling system (ProScan, Nanofinder) to determine the values of $h$ for the various values of $F$, the minimum required threshold fluence for discernible material removal, $F_{t}$, was determined for each laser (see Figs. 1, 2, 3 and 4) and is given in Table 2. Although this approach has hitherto been employed to examine pulsed lasers (excimer) and shown to be sound [27], the technique has also been used to investigate continuous wave (CW) lasers [16, 23-25]. What is more, the distinct linearity of the data points for the $\mathrm{CO}_{2}$ and HPDLs in Figs. 13 further validates the use of this approach for the examination of $\mathrm{CW}$ lasers. It is important to note that this would assume a minimum conduction loss. However, the PSZ bio-ceramic has relatively low thermal conductivity of only $1.8 \mathrm{~W} / \mathrm{mK}$ [28], thus it is reasonable to assume that the laser power densities used were high enough and that the shots were short enough to minimise the thermal conduction loss.

Now, the term $1 / \alpha$ is the absorption length, so by rearranging (1) thus:

$$
\frac{1}{\alpha}=\frac{h}{\ln \left(\frac{F}{F_{t}}\right)}
$$

then it is possible to determine the length to which the beams of all four lasers are absorbed by the PSZ bio-ceramic. By introducing $h$ and the corresponding value of $F$ for each data point into (2) it was possible to calculate the average absorption length for each laser under the actual experimental conditions (see Table 2). From a comparison of these absorption length values with the wavelengths at which each of the four laser emits given in Table 1, one can see that the absorption length appears to decrease with decreasing laser wavelength. It is worth remarking, however, that this decease is disproportionate to actual laser wavelength. 
It is interesting at this point to consider the actual absorptivity value of the surface of PSZ bioceramic. Absorptivity measurements made at room temperature using a comparative technique detailed elsewhere [29] revealed that the surface of the PSZ bio-ceramic absorbed around $68 \%$ of $\mathrm{CO}_{2}$ laser radiation, around $63 \%$ of the Nd:YAG laser radiation, around $59 \%$ of the HPDL radiation and around $53 \%$ of the excimer laser radiation. Now, for thick materials the sum of the absorptivity, $A$, and the reflectivity, $R$, is one. Accordingly $A$ is related to $R$, which in turn depends upon the (complex) refractive index. These present results suggest that the real part of the index is dominant. So, since these figures are of a similar order, it can be said that correlation between the actual absorptivity of the PSZ bio-ceramic and the absorption length for each of the four lasers is not evident. Thus it is reasonable to assume that absorption length, rather than actual absorptivity, plays a greater role in material coupling.

Work Dyer et al $[30,31]$ has revealed that the surface energy density or thermal loading, $\gamma$, of a material increases in an almost linear manner until $F_{t}$ is attained. Beyond this point $\gamma$ becomes almost constant due to the rapid ablation of products which carry away excess energy and consequently limit the maximum temperature rise. The value of $\gamma$ necessary to bring about removal is given by [31]

$$
\gamma=F_{t} \alpha
$$

From a rearrangement of (2) it is possible to calculate the value of the absorption coefficient, $\alpha$, for each of the four lasers. So, by introducing the values of $F_{t}$ for each of the four lasers deduced earlier from Figs. 1, 2, 3 and 4, along with the values of $\alpha$ for each laser into (3), then $\gamma$ for the $\mathrm{CO}_{2}$ laser, the Nd:YAG laser, the HPDL and the excimer laser were calculated (see Table 2).

\section{Conclusions}

Through the utilisation of a derivative of Beer-Lambert's law, the laser beam absorption lengths of $\mathrm{CO}_{2}$ laser, Nd:YAG laser, HPDL and excimer laser radiation for a PSZ bio-ceramic were determined. The absorption lengths, $1 / \alpha$, were calculated as $33.55 \times 10^{-3} \mathrm{~cm}$ for the $\mathrm{CO}_{2}$ laser, $18.22 \times 10^{-3} \mathrm{~cm}$ for the Nd:YAG laser, $17.17 \times 10^{-3} \mathrm{~cm}$ for the HPDL and $8.41 \times 10^{-6} \mathrm{~cm}$ for the excimer laser. The fluence threshold values at which significant material removal occurs, $F_{t}$, for the $\mathrm{CO}_{2}$ laser, Nd:YAG laser, HPDL and excimer laser were found to be $52 \mathrm{~J} / \mathrm{cm}^{2}, 97 \mathrm{~J} / \mathrm{cm}^{2}, 115 \mathrm{~J} / \mathrm{cm}^{2}$ and $0.48 \mathrm{~J} / \mathrm{cm}^{2}$ respectively. The thermal loading, $\gamma$, value for the $\mathrm{CO}_{2}$ laser, the Nd:YAG laser, the HPDL and the 
excimer laser were determined as $1.55 \mathrm{~kJ} / \mathrm{cm}^{3}, 5.32 \mathrm{~kJ} / \mathrm{cm}^{3}, 6.69 \mathrm{~kJ} / \mathrm{cm}^{3}$ and $57.04 \mathrm{~kJ} / \mathrm{cm}^{3}$ respectively. Correlation between the actual absorptivity of the PSZ bio-ceramic and the absorption length for each of the four lasers was not evident. Thus it is reasonable to assume that absorption length, rather than actual absorptivity, plays a greater role in material coupling. 


\section{REFERENCES}

1. F. Villermaux, M. Tabrizian, L.H. Meunier and D.L. Piron, Appl. Surf. Sci. 110, 62 (1997).

2. G.A. Guzzardella, P. Torricelli, N.N. Aldini and R. Giardino, Int. J. Artificial Organs, 24, 898 (2001).

3. J. Lawrence and L. Li, Mater. Sci. Eng. A, 303, 142 (2001).

4. J. Lawrence and L. Li, Proc. IMechE, B, 215, 1735 (2001).

5. P.G. Steg, R. Astier, J. Englander, A. Lavergne and D. Lencarpen, Archives des Maladies du Coeur des Vaisseaux, 4, 558 (1986).

6. T. Miki, K. Inoue, A. Obana and K. Shiraki, Lasers in Surgery and Medicine, 9, 543 (1989).

7. K.T. Schomaker, Y. Domankevitz, T.J. Flotte and T.F. Deutsch, Lasers in Surgery and Medicine, $11,141(1991)$.

8. M.J. Tassignon, N. Stempels, J. Nguyenlegros and F. Dewilde, Clinical and Experimental Ophthalmology, 229, 380 (1991).

9. C. Canning, P. Polkinhorne, A. Ariffen and Z. Gregor, Brit. J. Ophthalmology, 75, 602 (1991).

10. T.A. McMillan, W.C. Stewart, M.J. Nutaitis and T.P. Powers, Acta Opthalmologica, 70, 758 (1992)

11. D.R. Wyman, S.W. Schatz and J.A.C. Maguire, 1997 Lasers in Surgery and Medicine, 21, 50 (1997).

12. F. Dausinger, in Proceedings of ECLAT'90: Laser Treatment of Materials, Erlangen, Germany, SPIE, Bellingham (1991) Vol 1, pp. 1-14.

13. S. Shuttleworth, Appl. Surf. Sci. 96-98, 513 (1996).

14. P.E. Dyer, J. Gonzalo, P.E. Key, D. Sands and M.J.J. Schmidt, Appl. Surf. Sci. 110, 345 (1997).

15. X. Chen, W.T. Lotshaw, A.L. Ortiz, P.R. Staver and C.E. Erikson, J. of Laser Apps. 8, 233 (1996).

16. M.J.J. Schmidt, L. Li and J.T. Spencer, Appl. Surf. Sci. 138-139, 378 (1998).

17. L. Bradley, L. Li and F.H. Stott, Appl. Surf. Sci. 138-139, 522 (1998). 
18. J. Lawrence and L. Li, J. Phys. D, 32, 1075 (1999).

19. J. Lawrence and L. Li, J. Phys. D, 32, 2311 (1999).

20. J. Lawrence and L. Li, Appl. Surf. Sci. 154/155, 664 (2000).

21. J. Lawrence and L. Li, Mater. Sci. Eng. A, 284, 93 (2000).

22. J. Lawrence and L. Li, Mater. Sci. Eng. A, 287, 25 (2000).

23. J. Lawrence and L. Li, Optics Laser Tech. 32, 11 (2000).

24. J. Lawrence, E.P. Johnston and L. Li, J. Phys. D, 33, 745 (2000).

25. J. Lawrence and L. Li, Appl. Surf. Sci. 168, 71 (2000).

26. D. Bäuerle, Laser Processing and Chemistry, Springer, Berlin (1996).

27. J.E. Andrew, P.E. Dyer, D. Forster and P.E. Key, Appl. Phys. Lett. 43, 717 (1983).

28. A. Ravaglioli, Bioceramics: Materials, Properties and Applications, Chapman \& Hall, London (1992).

29. J. Lawrence, PhD Thesis, UMIST (1999).

30. P.E. Dyer and J. Sidhu, J. Appl. Phys. 57, 1420 (1985).

31. P.E. Dyer, S.D. Jenkins and J. Sidhu, Appl. Phys. Lett. 49, 453 (1986). 


\section{List of Figs.}

Fig. 1. Removal rate per shot, $h$, as a function of $\mathrm{CO}_{2}$ laser fluence, $F$.

Fig. 2. Removal rate per shot, $h$, as a function of Nd:YAG laser fluence, $F$.

Fig. 3. Removal rate per shot, $h$, as a function of HPDL fluence, $F$.

Fig. 4. Removal rate per shot, $h$, as a function of excimer laser fluence, $F$. 
Fig. 1

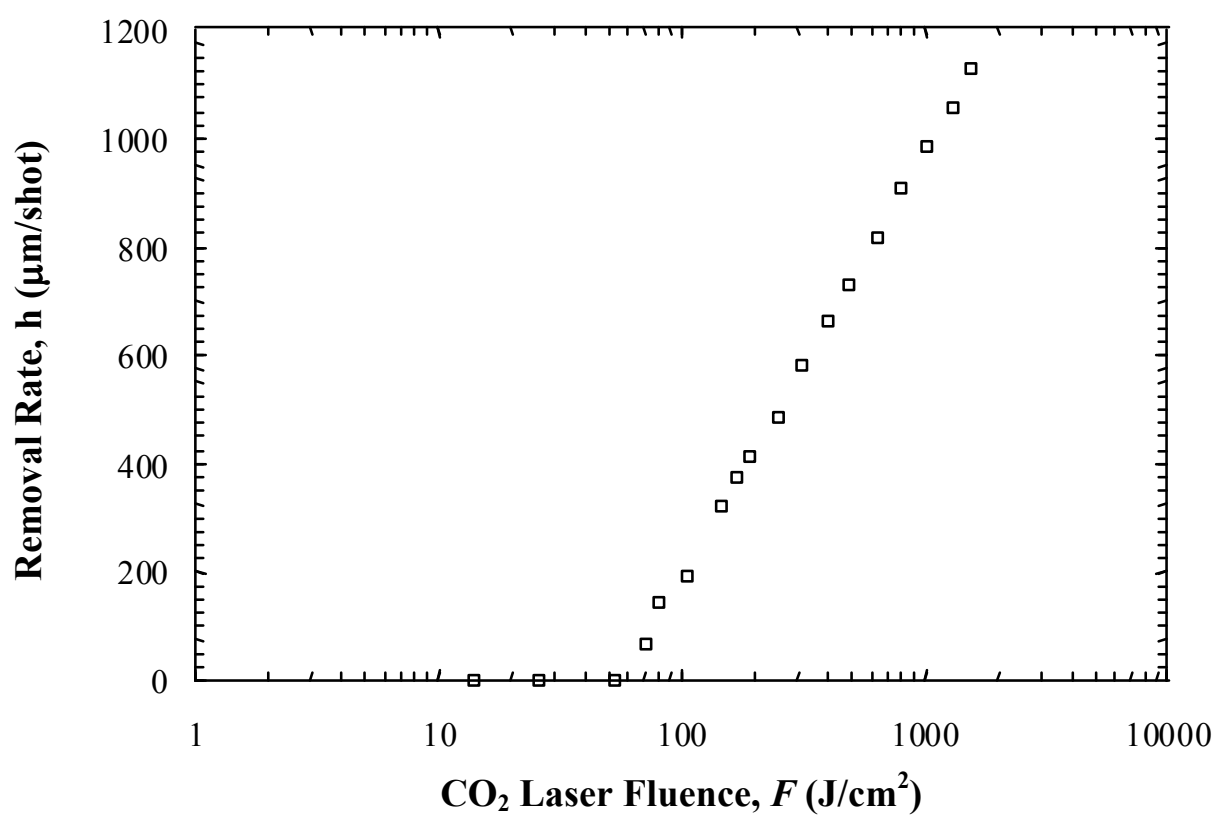


Fig. 2

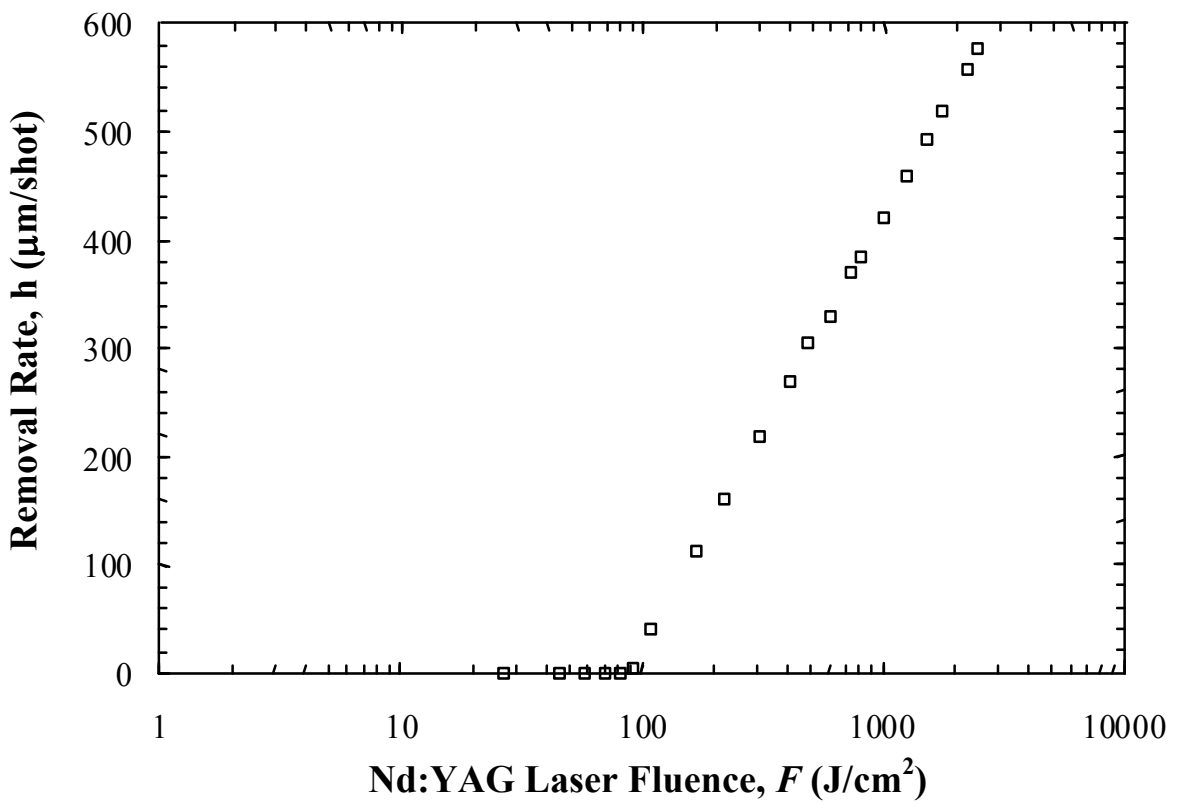


Fig. 3

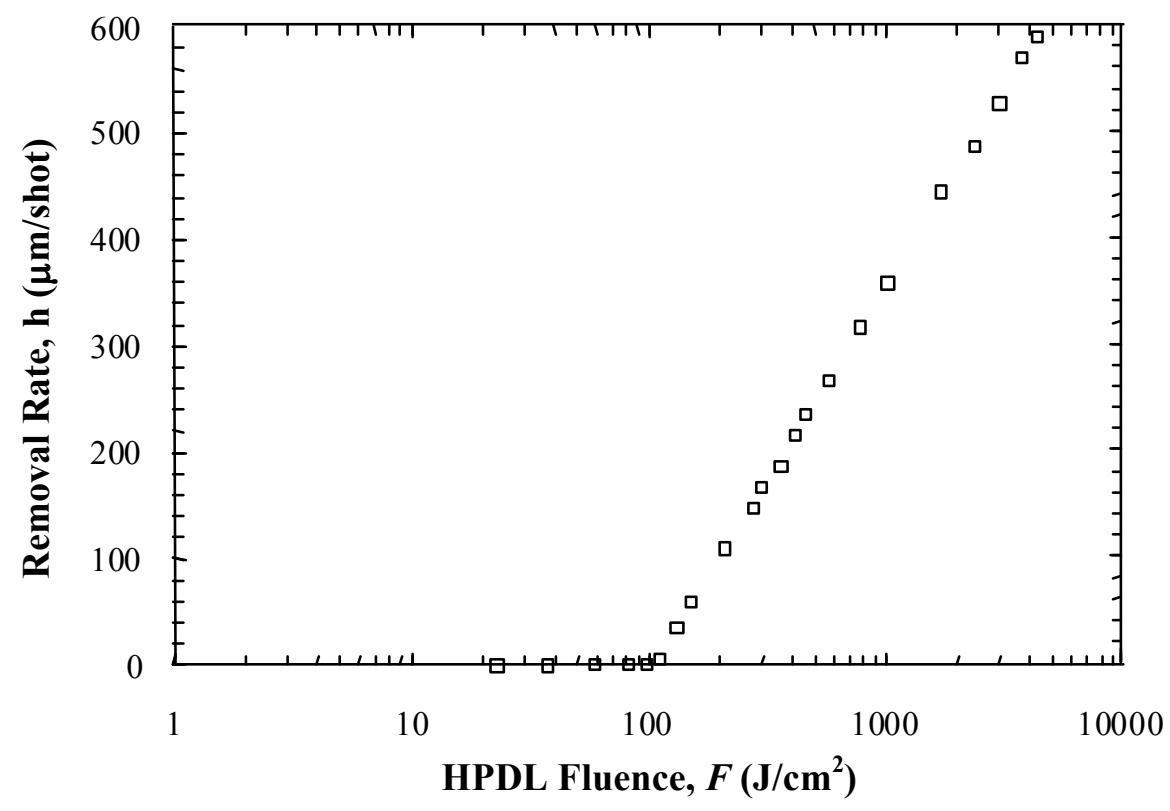


Fig. 4

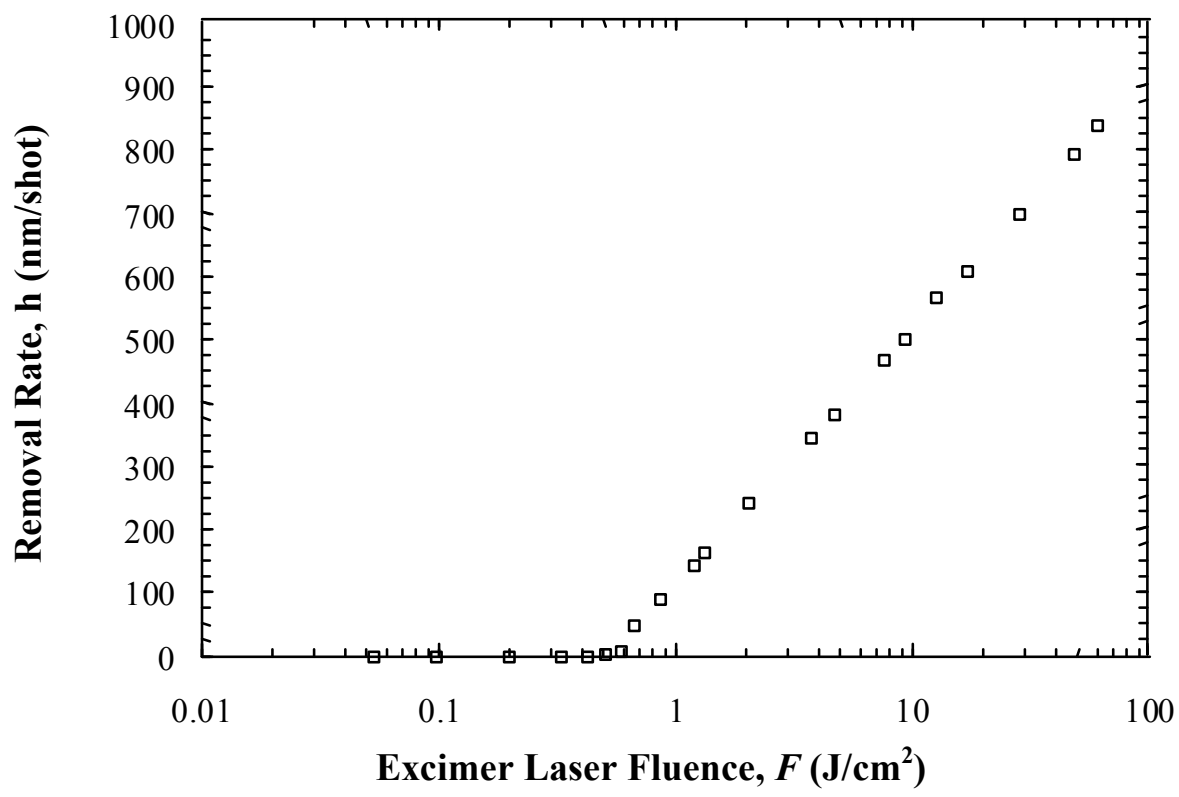




\section{List of Tables}

Table 1. Details of the selected industrial lasers used.

Table 2. Laser interaction properties and effects for the PSZ bio-ceramic. 
Table 1

\begin{tabular}{lcccc}
\hline \multirow{2}{*}{ Operating Characteristic } & \multicolumn{4}{c}{ Laser Type } \\
& $\mathbf{C O}_{2}$ & Nd:YAG & HPDL & Excimer \\
\hline Lasant & $\mathrm{CO}_{2}$ gas & Nd:YAG crystal & GaAlAs & KrF gas \\
Wavelength & $10.6 \mu \mathrm{m}$ & $1.06 \mu \mathrm{m}$ & $940 \pm 10 \mathrm{~nm}$ & $248 \mathrm{~nm}$ \\
Maximum average output & $3 \mathrm{~kW}$ & $400 \mathrm{~W}$ & $1.5 \mathrm{~kW}$ & $5 \mathrm{~W}$ \\
Maximum pulse energy & $\sim$ & $55 \mathrm{~J}$ & $\sim$ & $35 \mathrm{~J}$ \\
Pulse width & $\sim$ & $0.3-20 \mathrm{~ms}$ & $\sim$ & $20 \mathrm{~ns}$ \\
Repetition rate & $\sim$ & $0.2-500 \mathrm{~Hz}$ & $\sim$ & $1-55 \mathrm{~Hz}$ \\
Fibre core diameter & $\sim$ & $1 \mathrm{~mm}$ & $1 \mathrm{~mm}$ & $\sim$ \\
Mode of operation & $\mathrm{CW}$ & Pulsed (rapid) & $\mathrm{CW}$ & Pulsed (multiple) \\
\hline
\end{tabular}


Table 2

\begin{tabular}{lcccc}
\hline \multirow{1}{c}{ Value } & $\mathbf{C O}_{2}$ & Nd:YAG & HPDL & Excimer \\
\hline Threshold fluence, $F_{t}$ & $52 \mathrm{~J} / \mathrm{cm}^{2}$ & $97 \mathrm{~J} / \mathrm{cm}^{2}$ & $115 \mathrm{~J} / \mathrm{cm}^{2}$ & $0.48 \mathrm{~J} / \mathrm{cm}^{2}$ \\
Absorption length, $1 / \alpha$ & $33.55 \times 10^{-3} \mathrm{~cm}$ & $18.22 \times 10^{-3} \mathrm{~cm}$ & $17.17 \times 10^{-3} \mathrm{~cm}$ & $8.41 \times 10^{-6} \mathrm{~cm}$ \\
Absorption coefficient, $\alpha$ & $29.81 \mathrm{~cm}^{-1}$ & $54.88 \mathrm{~cm}^{-1}$ & $58.22 \mathrm{~cm}^{-1}$ & $118825.21 \mathrm{~cm}^{-1}$ \\
Absorptivity, $A$ & $68 \%$ & $63 \%$ & $59 \%$ & $53 \%$ \\
Thermal loading, $\gamma$ & $1.55 \mathrm{~kJ} / \mathrm{cm}^{3}$ & $5.32 \mathrm{~kJ} / \mathrm{cm}^{3}$ & $6.69 \mathrm{~kJ} / \mathrm{cm}^{3}$ & $57.04 \mathrm{~kJ} / \mathrm{cm}^{3}$ \\
\hline
\end{tabular}

